\title{
Management of late onset recurrent ventricular tachycardia following circumflex artery injury during minimally invasive mitral valve replacement surgery - Clinical case report
}

\author{
Timea Magdolna Szabo ${ }^{1,2^{*}}$, Mihaela Ispas ${ }^{1}$, Ayman Elkahlout ${ }^{1}$ \\ 1. Emergency Institute for Cardiovascular Diseases and Transplantation, Targu Mures, Romania \\ 2. Pharmaceutical biochemistry and the chemistry of environmental factors, George Emil Palade University of Medicine, Pharmacy, Science, and Technology of \\ Targu Mures, Romania
}

\begin{abstract}
Introduction: A rare complication of mitral valve surgery is the injury of the circumflex artery due to their close anatomical proximity resulting in a perioperative myocardial infarction and subsequent fibrosis with increased risk of developing ventricular arrhythmias. Case presentation: We hereby describe the case of a 74-year-old male patient who underwent minimally invasive mitral valve replacement surgery for severe mitral regurgitation two weeks prior to presenting to the emergency department with dyspnea, palpitations and slight thoracic discomfort. He was diagnosed with recurrent sustained monomorphic ventricular tachycardia due to inferior wall myocardial infarction. Angioplasty of the culprit lesion was attempted, but the procedure failed due to the elastic recoil of the vessel. Our patient received antiarrhythmic therapy and an implantable cardioverter-defibrillator for secondary prevention of sudden cardiac death, with no further recorded episodes of ventricular tachycardia. Conclusion: Although injury of the circumflex coronary artery during mitral valve surgery is described as rare, we strongly believe that increasing awareness of the potential risks involved can further prevent fatal complications.
\end{abstract}

Keywords: mitral valve bioprosthesis, ventricular tachycardia, myocardial infarction, circumflex iatrogenic injury

Received 15 January 2021 / Accepted 21 March 2021

\section{Introduction}

Surgery represents the first-line treatment for severe chronic primary mitral regurgitation in symptomatic patients. Mitral valve repair surgery is recommended when possible. Mitral valve replacement surgery with preservation of the subvalvular apparatus represents the second-best choice when mitral valve repair is not feasible [1].

Minimally invasive mitral valve surgery represents a novel alternative to the median sternotomy method in several specialized cardiothoracic surgery centers. The technique of minimally invasive mitral valve surgery has improved considerably in the past 24 years since it was first performed, and it is associated with favorable short- and long-term outcomes, currently representing a safe and beneficial option for selected patients, although the conventional approach remains the gold standard [2].

The iatrogenic injury of the left circumflex artery is unanimously accepted as an intraoperative complication in both approaches - minimally invasive, through right lateral minithoracotomy, or conventional, through median sternotomy. The injury of the circumflex artery may occur due to the anatomical relationship between the vessel and the posterior mitral valve annulus localized in close vicinity to the anterolateral commissure, especially when patients present left-dominant or balanced coronary network [3].

* Correspondence to: Timea Magdolna Szabo

E-mail: szabotimeamagdolna@gmail.com
In this paper we report the case of an elderly patient, who developed life-threatening ventricular arrhythmias due to perioperative circumflex coronary artery injury sustained during mitral valve surgery, and who received specific antiarrhythmic treatment. A signed consent for the publication of this case was obtained from the patient.

\section{Case presentation}

A 74-year-old male patient presented to the emergency department complaining of dyspnea, palpitations and slight thoracic discomfort. The patient's recent medical history included minimally invasive mitral valve replacement surgery performed two weeks prior through right lateral minithoracotomy. The indication for surgery was severe degenerative mitral valve regurgitation due to anterior leaflet prolapse (A1, A2 segments), and he received a Hancock II bioprosthetic mitral valve size 31 . The preoperative cardiac catheterization showed normal coronary arteries. The patient had been previously diagnosed with paroxysmal atrial fibrillation and at the time of evaluation was on oral anticoagulant therapy with a vitamin $\mathrm{K}$ antagonist, but with an INR below therapeutic range. Physical examination revealed the following abnormal findings: a blood pressure reading of $90 / 55 \mathrm{mmHg}$, a regular heart rate of $190 \mathrm{bpm}$, cold extremities and diaphoresis. As part of the initial diagnostic workup, an electrocardiogram was performed, which showed sustained monomorphic ventricu- 
lar tachycardia. Lidocaine, then amiodarone was given as i.v. bolus therapy, but the arrhythmia persisted. Due to decreasing blood pressure values, electrical cardioversion with prior sedation was performed, requiring two escalating energy sequence of 150 -joule and 200-joule biphasic shocks, as the first one was inefficient. After cardioversion sinus rhythm was restored, but with a transient third-degree atrioventricular block. Another 12-lead electrocardiogram was recorded, describing a heart rate of $70 \mathrm{bpm}$ and the following abnormal findings: PQ interval of $200 \mathrm{msec}$, Q-waves with $1 \mathrm{~mm}$ ST-segment elevation in DIII and downsloping or horizontal 1-2 mm ST-segment depression in leads V4-V6, DI, aVL. Posterior and right-sided precordial lead electrocardiograms were also obtained showing no evidence of myocardial ischemia (Figure 1).

Due to this conduction abnormality, the decision to administer further i.v. amiodarone was therefore delayed. High-sensitivity cardiac troponin levels were indicative of myocardial injury. Transthoracic echocardiography showed a dilated left ventricle with mild hypertrophy, an ejection fraction of $40 \%$ caused by posteroinferior wall akinesia and lateral wall hypokinesia, alongside a normally functioning bioprosthetic mitral valve. Urgent coronary angiography was performed which revealed left coronary dominance and proximal circumflex artery occlusion localized in close anatomical proximity to the suturing ring of the bioprosthetic mitral valve. No other significant coronary stenoses were identified. Percutaneous coronary intervention (PCI) with stenting of the culprit vessel was attempted, but the procedure was abandoned due to the difficult insertion of the angioplasty guidewire and balloon catheter, and elastic recoil of the arterial segment even after inflating a $2.0 \mathrm{~mm}$ balloon to $10 \mathrm{~atm}$ pressure. Intracoronary imaging was not available for the aforementioned reasons. Manual thrombus aspiration was also performed twice, but no thrombotic material was extracted (Figure 2).

Following the procedure the patient was admitted to the coronary care unit where his cardiac rhythm and vital signs were closely monitored. Five hours later the patient presented another episode of sustained ventricular tachycardia which again required sedation and electrical cardioversion. Despite the persistent prolonged atrioventricular conduction and transient complete heart block, the decision to administer i.v. amiodarone was taken in-order-to prevent recurrent arrhythmias. Repeated measurements of cardiac troponin levels were not performed. One week prior to discharge the patient received a Biotronik Iforia (Biotronik Inc, OR, USA) 5 DR-T pro MRI dual-chamber implantable cardiac defibrillator (ICD) for secondary prevention of sudden cardiac death. Transthoracic echocardiography follow-up during admission revealed persistent inferior wall motion abnormalities. No further arrhythmias, conduction abnormalities or hemodynamic instability were

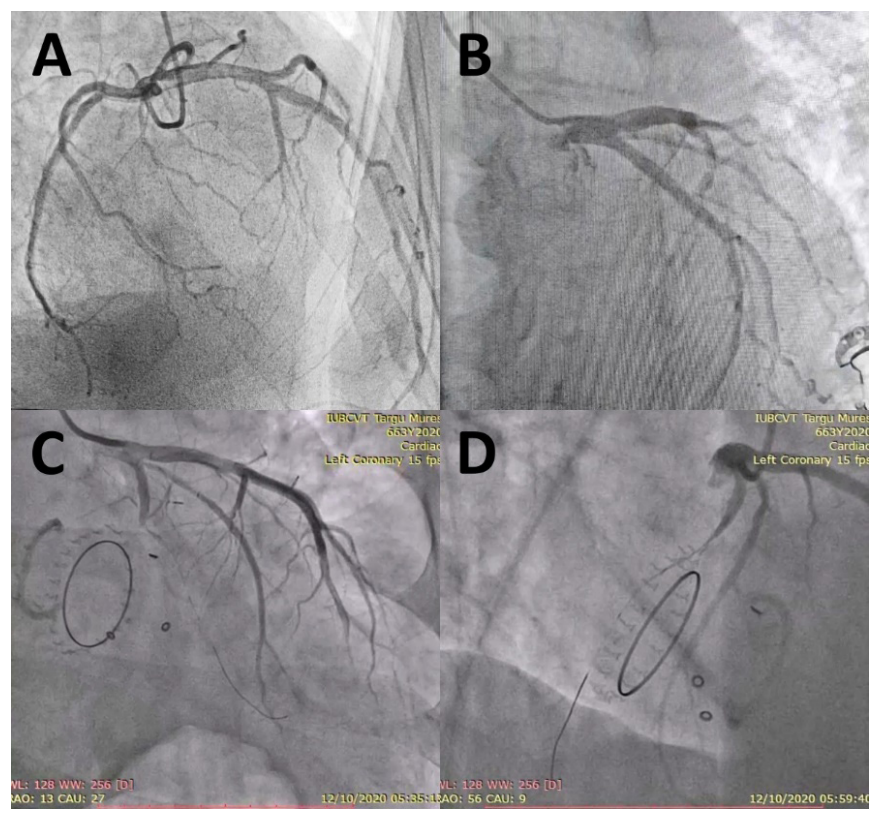

Fig. 2. Coronary angiogram; A,B, The preoperative coronary angiogram showing normal coronary arteries. C,D, Images showing proximal left circumflex artery occlusion localized in the vicinity of the suturing ring of the bioprosthetic mitral valve. Results after repeated balloon inflation at the site of the culprit lesion.
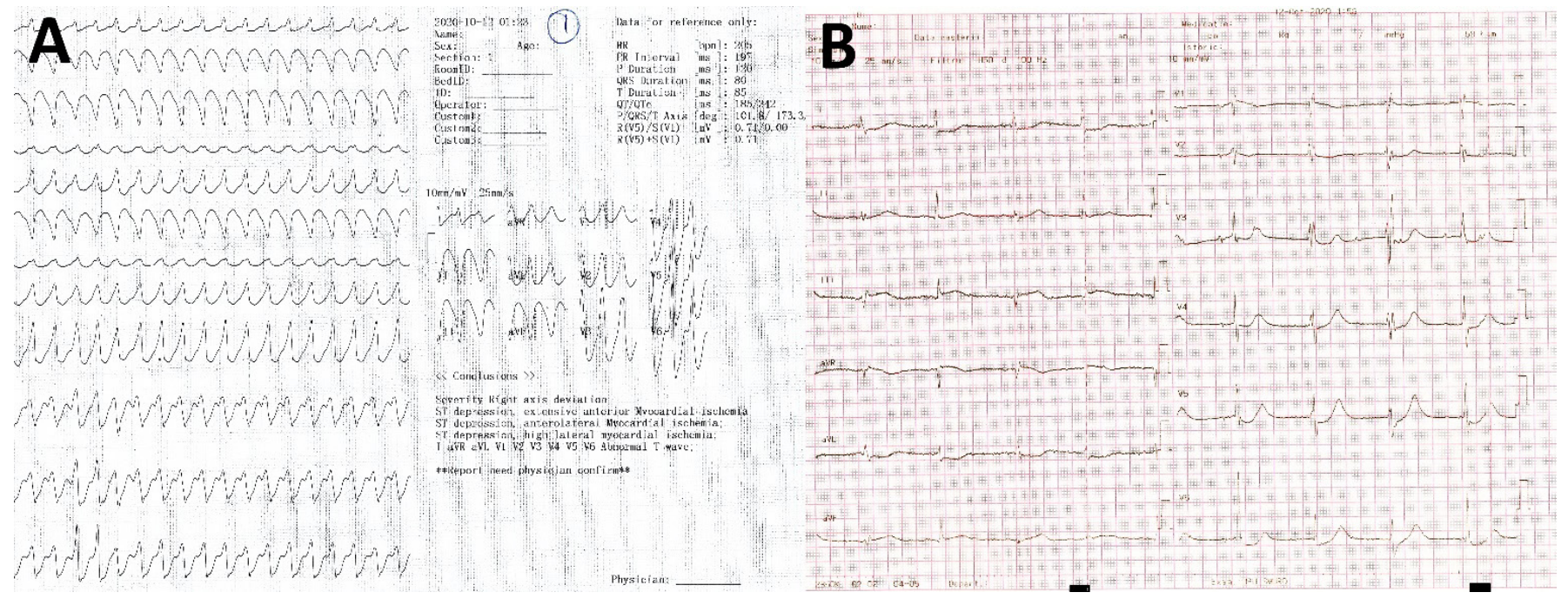

Fig. 1. Electrocardiogram; A, Sustained monomorphic ventricular tachycardia at presentation. B, Third degree atrioventricular block after electrical cardioversion. 
recorded during hospitalization and the patient was discharged on antiarrhythmic drug treatment, low dose aspirin, vitamin $\mathrm{K}$ antagonist, low dose betablocker, angiotensin-converting enzyme inhibitor, statin and diuretic medication.

\section{Discussions}

The clinical presentation of myocardial ischemia due to epicardial coronary injury during mitral valve surgery varies significantly, and depends on the site of the arterial occlusion, the ventricular systolic function and pattern of injury - partial, complete or dynamic $[4,5]$. Ischemia develops most frequently during surgery or in the immediate postoperative period. Late presentations are less common, they are described in only $14 \%$ of selected cases, and may develop ventricular arrhythmias [6]. We suspect that due to postoperative analgesia and frequently non-diagnostic electrocardiographic findings regarding left circumflex occlusion, myocardial ischemia may remain sometimes unrecognized, especially if the jeopardized myocardium area is small. This may result in myocardial scar development and interstitial fibrosis exposing the patient to an increased risk of developing ventricular arrhythmias.

There are several mechanisms described through which injury of the left circumflex coronary artery may occur: arterial distortion, kinking of the vessel due to tissue retraction causing dynamic or fixed occlusion, external compression of the artery due to local hematoma or the bioprosthetic valve ring, encircling of the vessel by the fixation suture, endothelial laceration causing intraluminal thrombosis or coronary spasm [6]. The most likely etiology of the arterial occlusion in our patient was the intraoperative fixation of the artery by the surrounding suture, which is evidenced by the difficult insertion of the angioplasty guidewire and balloon catheter and the elastic recoil of the arterial segment after balloon deflation.

The preferred therapeutic management strategy of left circumflex artery injury due to mitral valve surgery is interventional, especially when patients are hemodynamically stable, or the myocardial ischemia has a late onset $[4,5]$. When hemodynamic instability due to injury of the epicardial arteries occurs during surgery, a revision of the mitral valve or concomitant coronary artery bypass grafting are the two most suitable options $[4,5]$. Atrioventricular sulcus rupture may complicate the mitral valve replacement surgery especially in the elderly [7], therefore a surgical reintervention would expose the patient to a similar risk. A possible complication of PCI with stent implantation may be coronary to left atrium fistula formation due to the frailty of the mitral valve periannular tissue. Other complications may arise by using both procedures, and the proper management of circumflex artery injury is not yet well established [8]. Because our patient presented with a complex circumflex artery lesion that could not be repaired by conventional percutaneous approach, the risk of recurrent arrhythmias was considered significant, and the decision to implant an ICD for secondary prevention of sudden cardiac death was made. Catheter ablation of post-myocardial infarction ventricular tachycardia was not considered feasible due to the presence of the mitral bioprosthesis. Betablocker therapy in combination with amiodarone was also initiated in-order-to reduce the number of ICD shocks, in accordance to the current European Society of Cardiology guidelines [9].

\section{Conclusions}

The appropriate therapeutical management of a complex clinical case requires an interdisciplinary approach. Epicardial coronary artery lesions may occur during mitral valve surgery. This potentially life-threatening complication can manifest even after the critical postoperative period and should be immediately recognized in order to reduce morbidity and prevent death.

\section{Aknowledgements}

We wish to thank Dr. Dan Nistor for the insightful comments and English language editing on our manuscript.

\section{Authors' contribution}

TMSZ (Patient follow-up; Conceptualization; Data collection and analyzing; Writing - original draft)

MI (Therapeutic management of the case; Conceptualization; Writing - review and editing)

AE (Therapeutic management of the case; Writing - review and editing)

\section{Conflict of interest}

None to declare

\section{References}

1. Baumgartner H, Falk V, Bax JJ et al. 2017 ESC/EACTS Guidelines for the management of valvular heart disease. Eur Heart J. 2017;38:2739-2791.

2. Kastengren M, Svenarud P, Ahlsson A, Dalén M. Minimally invasive mitral valve surgery is associated with a low rate of complications. J Intern Med. 2019;286:614-626.

3. Virmani R, Chun PK, Parker J, McAllister Jr HA. Suture obliteration of the circumflex coronary artery in three patients undergoing mitral valve operation. Role of left dominant or codominant coronary artery. J Thorac Cardiovasc Surg. 1982;84:773-778.

4. Grande AM, Fiore A, Massetti M, Viganò M. latrogenic Circumflex Coronary Lesion in Mitral Valve Surgery. Tex Heart Inst J. 2008;35:179183.

5. Coutinho GF, Leite F, Antunes MJ. Circumflex artery injury during mitral valve repair: Not well known, perhaps not so infrequent-lessons learned from a 6-case experience. J Thorac Cardiovasc Surg. 2017;154:16131620.

6. Hiltrop N, Bennett J, Desmet W. Circumflex coronary artery injury after mitral valve surgery: A report of four cases and comprehensive review of the literature. Catheter Cardiovasc Interv. 2017;89:78-92.

7. Kwon, JT, Jung TE, Lee DH. The rupture of atrioventricular groove after mitral valve replacement in an elderly patient. J Cardiothorac Surg. 2014;9:28.

8. Somekh NN, Haider A, Makaryus AN, Katz S, Bello S, Hartman A. Left circumflex coronary artery occlusion after mitral valve annuloplasty: "a stitch in time". Tex Heart Inst J. 2012;39:104-107.

9. Priori SG, Blomström-Lundqvist C, Mazzanti A et al. 2015 ESC Guidelines for the management of patients with ventricular arrhythmias and the prevention of sudden cardiac death. Eur Heart J. 2015;36: 2793-2867. 